\title{
Angular instability due to radiation pressure in the LIGO gravitational-wave detector
}

\author{
Eiichi Hirose,${ }^{1,4, *}$ Keita Kawabe, ${ }^{2}$ Daniel Sigg, ${ }^{2}$ Rana Adhikari, ${ }^{3}$ and Peter R. Saulson ${ }^{1}$ \\ 'Department of Physics, Syracuse University, Syracuse, New York 13244, USA \\ ${ }^{2}$ Laser Interferometer Gravitational-Wave Observatory (LIGO) Hanford Observatory, \\ 127124 North Route 10, Richland, Washington 99354, USA \\ ${ }^{3}$ LIGO Laboratory, California Institute of Technology, MS 18-34, Pasadena, California 91125, USA \\ ${ }^{4}$ Currently with Harvard-Smithsonian Center for Astrophysics, 60 Garden Street MS 50, \\ Cambridge, Massachusetts 02138, USA \\ *Corresponding author: eiich.hirose@gmail.com
}

Received 3 September 2009; revised 10 December 2009; accepted 10 May 2010; posted 11 May 2010 (Doc. ID 116355); published 11 June 2010

\begin{abstract}
We observed the effect of radiation pressure on the angular sensing and control system of the Laser Interferometer Gravitational-Wave Observatory (LIGO) interferometer's core optics at LIGO Hanford Observatory. This is the first measurement of this effect in a complete gravitational-wave interferometer. Only one of the two angular modes survives with feedback control, because the other mode is suppressed when the control gain is sufficiently large. We developed a mathematical model to understand the physics of the system. This model matches well with the dynamics that we observe. (C) 2010 Optical Society of America

OCIS codes: $\quad 000.2780,120.3180,120.2230,220.1140$.
\end{abstract}

\section{Gravitational-Wave Interferometers}

Gravitational-wave detection technology has made great strides in recent years, and more progress is expected in the near future. In the U.S., the LIGO project has constructed several large interferometers, two at Hanford, Washington, and another one at Livingston, Louisiana [1,2]. These initial LIGO interferometers were used to make observations of unprecedented sensitivity during the period November 2005 through September 2007, LIGO's fifth science run, during which one year's worth of triple coincident data were collected at an rms strain sensitivity of about $10^{-21}$ [3-5]. The LIGO Scientific Collaboration is now completing the analysis of these data. Operation of LIGO and analysis of gravitational-wave data are closely coordinated with two European projects, GEO (a British-German collaboration with a

0003-6935/10/183474-11\$15.00/0

(C) 2010 Optical Society of America
$0.6 \mathrm{~km}$ interferometer near Hannover [6]) and VIRGO (a French-Italian collaboration with a $3 \mathrm{~km}$ interferometer near Pisa [7]).

As striking as this technological progress is, it is widely expected that sensitivity improvement of about a factor of 10 in strain amplitude will be required before observations of gravitational waves become routine. Fortunately, the technology to make the required improvement is at hand. For LIGO, those improvements will be embodied in Advanced LIGO, now under construction and expected to begin operations around 2015 [8]. In the meantime, one additional data run is being carried out with improved initial LIGO hardware. This incremental improvement, called Enhanced LIGO, has already started its science run.

The challenge of gravitational-wave detection is that the signals are tiny-differential strains in two perpendicular direction with amplitudes below $10^{-21}$. A Michelson interferometer is well suited to detecting a differential strain, but it needs extraordinary sensitivity. LIGO has addressed the sensitivity as follows 
$[1,2]$ (other interferometers have solutions that are similar in most features but differ in details): LIGO's interferometers have arms that are $4 \mathrm{~km}$ in length (the second of the two interferometers at LIGO Hanford Observatory is only $2 \mathrm{~km}$ long). The $10 \mathrm{~kg}$ mirrors are suspended as carefully engineered pendulums, which are in turn connected to multistage seismic isolation systems. A high-power Nd:YAG laser (with power of $10 \mathrm{~W}$ ) is used to provide sufficient optical power to reduce the phase noise due to photon shot noise. The simple Michelson interferometer configuration is augmented by several features that substantially enhance efficiency. Each arm is made up of a Fabry-Perot cavity held on resonance, thus amplifying the phase shift by a factor of $\mathcal{F} / \pi$ (the cavity's finesse $\mathcal{F}$ is about 200). The effective laser power is enhanced by an additional factor of about 50 by placing a partly transmitting mirror between the laser and the rest of the interferometer; the combination of this recycling mirror and the rest of the interferometer forms an additional Fabry-Perot cavity (power recycling cavity) that is held on resonance [9]. The whole system is enclosed in a vacuum system held at $10^{-8}$ Torr [see the schematic diagram in Fig. 6]. The use of such high laser power levels in resonant cavities means that radiation pressure will play an important role in the operation of LIGO.

\section{Role of Radiation Pressure in Gravitational-Wave Detectors}

To understand the dynamics of the interferometers, we need to examine the power levels of the actual LIGO design. Light arrives at the recycling mirror with a power of $5 \mathrm{~W}$, but, with the power recycling mirror held at the resonance condition, inboard of the recycling mirror the effective power level hitting the interferometer's beam splitter is $250 \mathrm{~W}$. Inside the resonant cavities that make up each of the long Michelson arms, the power level is $12 \mathrm{~kW}$. Thus, the radiation pressure on each of the arm mirrors is $10^{-4} \mathrm{~N}$. Incident on a $10 \mathrm{~kg}$ mirror held in a pendulum suspension with resonant frequency of $0.5 \mathrm{~Hz}$, this force would cause a displacement of $10^{-6} \mathrm{~m}$. Of course, a Michelson interferometer with suspended mirrors only functions properly with a control system to hold the mirrors at the proper longitudinal position and alignment; this control system acts to counteract the radiation pressure force. A coupling of radiation pressure to longitudinal motion of the mirrors has been experimentally demonstrated by Corbitt et al. [10].

Sidles and Sigg [11], building on earlier work by Solimeno et al. [12], pointed out that torques due to radiation pressure can cause an interesting problem in interferometers with suspended mirrors. They showed that the angular coupling between the mirrors due to radiation pressure is best understood by considering the dynamics of a cavity as a set of normal modes. At large enough radiation pressures, one of these modes can become unstable. Although the LIGO detectors control not only longi- tudinal motion but also angular motion of the mirrors, the servo bandwidth cannot be arbitrarily turned up due to noise coupling into the gravitational-wave output. Therefore, we need to have a detailed understanding of the dynamics of this instability, in an interferometer with an angular control system.

In this paper, we first review the theory developed by Sidles and Sigg, giving a heuristic understanding of the instability that they predicted. We point out that initial LIGO operated successfully at power levels that would be expected to cause angular instability, if one made a naive application of their theory. In the next section, we present a measurement of the angular response to torque applied to a LIGO mirror, made under normal operating conditions (and at other laser power levels as well). The mirror response shows features different from those expected by the theory, as presented in Ref. [11].

The difference from the simple theory is not surprising, because a control system acts on the mirrors of the interferometer. In order to derive the expected response, we modeled the combined mechanicaloptical-control system using MATLAB and Simulink [13]. Section 5 of the paper describes this model, and shows how our measurements agree with this more complete theory of the dynamics of the system. In particular, we can now understand the stability of initial LIGO at power levels at which instability was expected in an uncontrolled interferometer. Our measurements show that, inside the control system, the mirror's angular transfer function is indeed that of an unstable harmonic oscillator.

In Section $\underline{6}$ of the paper, we investigate how much the laser power can be increased before the present control system would be overwhelmed by the radiation pressure instability.

\section{Review of Sidles-Sigg instability}

Consider an optical resonator that consists of two suspended mirrors and laser light, which resonates between the mirrors [Fig. 1]. The sketch shows two mirrors, which have very large radii of curvature (larger than the distance between two mirrors in LIGO detectors); the optical axis is the line connecting the centers of curvature of the mirrors. The two mirrors are subject to a natural restoring force that comes from the wire loop suspending them from the suspension system.

When the cavity is resonant, radiation pressure from the laser in the cavity can become important. With no light, each mirror exhibits independent torsional oscillations. When the cavity is filled with light, there are two coupled modes of the optomechanical system. This description can be applied either to the yaw mode or the pitch mode of the mirrors in the cavity. The measurements described below were made in the yaw mode.

Suppose that the mirrors oscillate in such a way that the signs of mirror angles $\theta_{1}, \theta_{2}$ are either $(+,-)$ or $(-,+)$. In this situation, radiation pressure works 


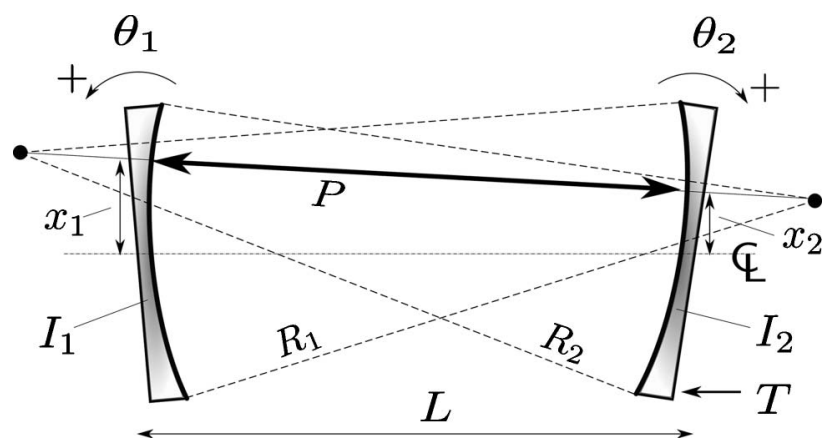

Fig. 1. Schematic diagram of two mirrors, coupled by radiation pressure caused by the power of the laser beam $P$ pushing at distances $x_{1}$ and $x_{2}$ away from the center line, when the mirrors are tilted at angles of $\theta_{1}$ and $\theta_{2}$, respectively. $I_{1}$ and $I_{2}$ are the moments of inertia of the mirrors. $R_{1}, R_{2}$, and $L$ are the radii of curvature of the two mirrors and the distance between the mirrors. $T$ is an external torque applied to one of the mirrors to study the system. The sketch can be viewed as having either yaw motion or pitch motion.

so as to enhance the original restoring force by pushing the mirrors back to the original position, as shown in Fig. 2(a), which means the mirrors experience a stronger restoring torque than that due to the mechanical restoring torque alone. Thus, the eigenfrequency of this mode will be higher than the original uncoupled pendulum natural frequency.

On the other hand, suppose that both mirrors tilt in such a way that the sign of mirror angle $\theta_{1}, \theta_{2}$ is either $(+,+)$ or $(-,-)$. In that situation, the radiation pressure works against the original restoring force. Tilt angles in this pattern cause the beam to move sideways, as shown in Fig. 2(b), which means that the net restoring force will be smaller than that

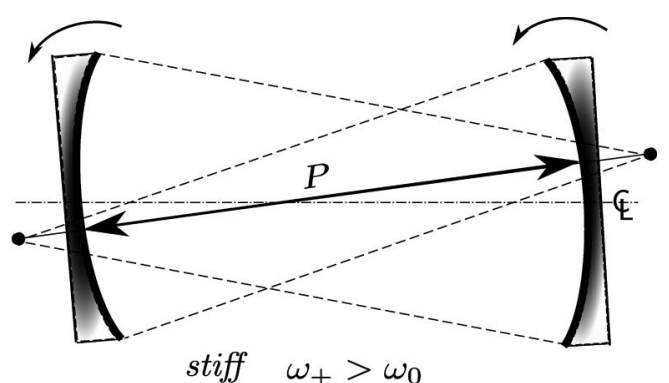

(a)

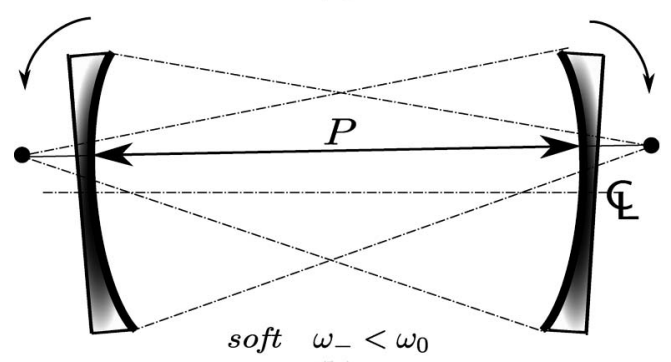

(b)

Fig. 2. (a) Stiff mode of angular motion in optical resonator. Radiation pressure works to enhance the mechanical restoring force. (b) Soft mode of angular motion in optical resonator. Radiation pressure works against the restoring force from the wire, and if the power exceeds a critical value, then this mode is unstable. due to the mechanical restoring force alone. When the power inside the optical resonator exceeds a critical value, the net restoring torque will become negative.

In order to investigate the dynamics of this system, we can apply an external torque to one of the mirrors and determine the transfer function of the mirror's angle to the applied torque. The normalized transfer function will be

$$
H(s)=\frac{\theta_{2}}{T} \sim \frac{-\left(s^{2}+\omega_{z}^{2}\right)}{\left(s^{2}+\omega_{-}^{2}\right)\left(s^{2}+\omega_{+}^{2}\right)} .
$$

The two pairs of poles $\omega_{ \pm}$and the pair of zeros $\omega_{z}$ are given by

$$
\begin{gathered}
\omega_{ \pm}^{2}=\omega_{0}^{2}+\frac{P L}{I c}\left[\frac{-\left(g_{1}+g_{2}\right) \pm \sqrt{4+\left(g_{1}-g_{2}\right)^{2}}}{1-g_{1} g_{2}}\right], \\
\omega_{z}^{2}=\omega_{0}^{2}-\frac{2 P L}{c I} \frac{g_{2}}{1-g_{1} g_{2}},
\end{gathered}
$$

where $\omega_{0}$ is the mechanical resonant frequency of the torsion pendulum, $P$ is the laser power inside the cavity, $L$ is the length of the cavity, $I$ is the moment of inertia of the mirror $\left(\sim 0.047 \mathrm{k} \mathrm{gm}^{2}\right)$, and $c$ is the speed of light. The factors $g_{1}$ and $g_{2}$ are the $g$ parameters of the cavity, defined by $g_{1,2}=1-L / R_{1,2}(0.71$, 0.45 , respectively) where $R_{1}$ and $R_{2}$ are the radii of curvature of the mirrors.

Here is an intuitive justification of the transfer function, which describes the process of shaking one of the mirrors and observing the response of that mirror. Because there are two angular modes of the coupled system, one will see resonances at the two different frequencies, which correspond to the two pairs of poles $\omega_{ \pm}$. Given the phase relations between these two modes, there will be a frequency at which the mirror does not move at all. This frequency corresponds to the pair of zeros $\omega_{z}$. If we observe the other mirror, however, the two modes have a different phase relationship, and the pair of zeros does not appear in that other transfer function.

As $P$, the laser power circulating inside the cavity, increases, $\omega_{+}$increases, while $\omega_{-}$and $\omega_{z}$ decrease [see Fig. 3]. Eventually, both $\omega_{-}^{2}$ and $\omega_{z}^{2}$ become negative, and the corresponding poles and zeros become real. $P_{1}$ and $P_{2}$ in Fig. 3 are the powers that give $\omega_{-}^{2}=$ 0 and $\omega_{z}^{2}=0$, respectively. For the initial LIGO, they are roughly $P_{1}=7.5 \mathrm{~kW}$ and $P_{2}=26.4 \mathrm{~kW}$. A set of Bode diagrams are shown in Fig. $\underline{4}$, and a pole map of Eq. (1) is shown in Fig. 5.

When $P=0$, the two mirrors are uncoupled. The transfer function must be that for a simple torsion pendulum. When $P$ is nonzero but sufficiently small, the two mirrors will be coupled and, thus, the Bode diagram will have two peaks associated with the two pairs of poles and one dip from the pair of zeros. In the $s$ plane, the two pairs of poles at $s= \pm i \omega_{0}$, denoted by triangles in Fig. 5 , split into nondegenerate 


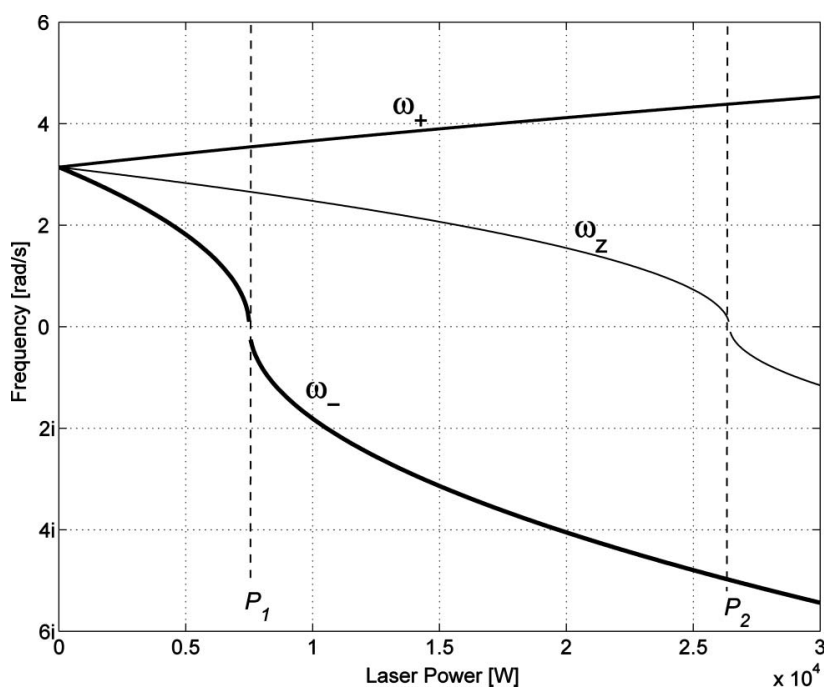

Fig. 3. Angular frequencies of poles and zeros as a function of cavity laser power, according to Eqs. (1) $-(3)$. As $P$ increases, $\omega_{+}$increases, while $\omega_{-}$and $\omega_{z}$ decrease as $P$ increases and become negative above $P_{1}$ and $P_{2}$, respectively.

pairs, one moving toward the origin and one moving away. When $P$ reaches $P_{1}$, the pair associated with $\omega_{-}$ reaches the origin, and for higher powers, those poles move along the real axis. When $P$ reaches $P_{2}$, above which $\omega_{z}^{2}$ becomes negative, the dip corresponding to $\omega_{z}$ will disappear from the Bode diagram. The filled circles in Fig. 5 are the poles at the operating laser power in initial LIGO. The existence of the pole in the right half-plane indicates instability. At $P>P_{1}$, the initial LIGO would be unstable in the absence of an angular control system.
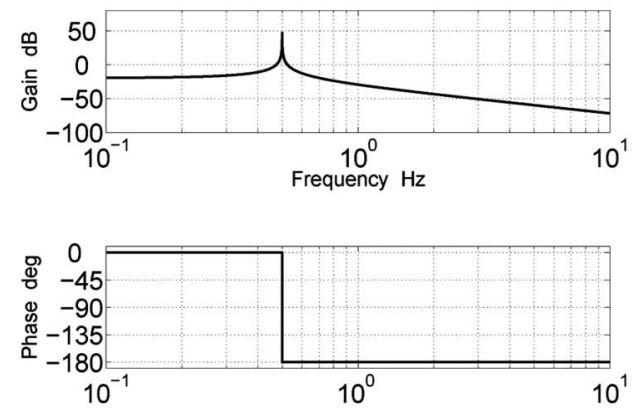

(a)
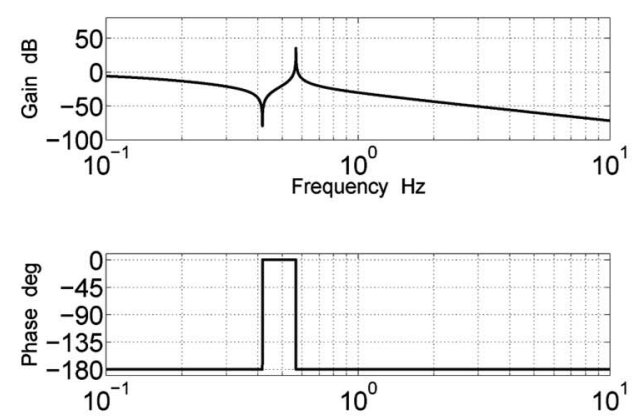

(c)

\section{Measurement}

To study the radiation pressure effect, we made a set of measurements on one of the arm cavities in the $4 \mathrm{~km}$ interferometer at LIGO Hanford Observatory. We wanted to study the regime $P>P_{1}$, as described above, so all control loops (including angular controls) were engaged during the measurement. The angular sensing and control system [14-17] has ten degrees of freedom. They are the pitch and yaw motion of the five core optics [the two pairs of mirrors: (ITMX, ETMX) and (ITMY, ETMY) in the long Fabry-Perot cavities and the power recycling mirror (RM) located upstream of the Michelson interferometer; see Fig. 6]. These degrees of freedom are measured by the quadrant photodetectors called wavefront sensors $[18,19]$ and are controlled by electromagnetic actuators attached to the backs of the mirrors. We reduce the ten degrees of freedom to two by looking at only two wavefront sensors, which are sensitive to the differential degrees of freedom (i.e., $\Delta \theta_{\mathrm{ETM}}=\theta_{2}-\theta_{4}$ and $\Delta \theta_{\mathrm{ITM}}=\theta_{1}-\theta_{3}$ ).

Besides these wavefront sensors for global angular sensing, there are sensors called optical levers [20], which work only locally. An optical lever consists of a diode laser and a quadrant photodiode, which senses the position of the laser spot reflected by the mirror and, thus, allows a measurement of the angle of the mirror. We used the optical lever signal to monitor the response of the mirror to angular excitation; this enabled us to measure the transfer function $H(s)$ that we investigated in the previous section.

In order to start the measurement, we first ensured that the interferometer was in its ordinary
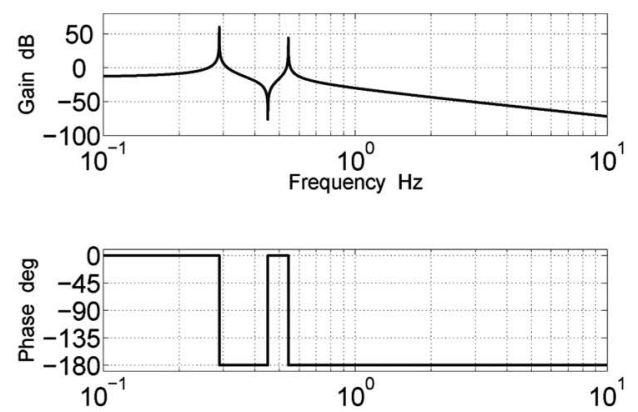

(b)
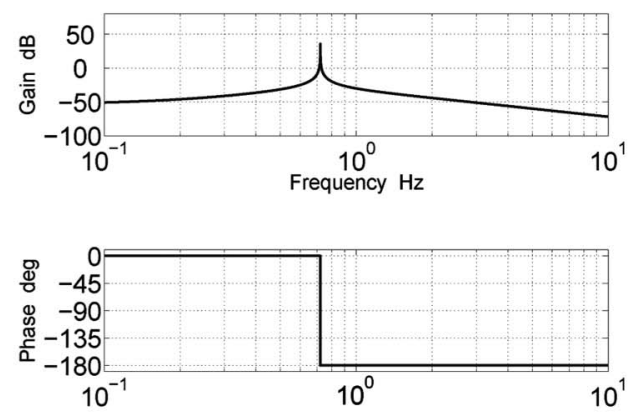

(d)

Fig. 4. Bode plot of the uncontrolled two-mirror system at various powers. (a) $P=0$, (b) $0<P<P_{1}$, (c) $P_{1}<P<P_{2}$, and (d) $P>P_{2}$. Note that the bottom two plots correspond to the unstable cases in which an external servo is necessary to keep the system stable. 
$\operatorname{Im}(\mathbf{s})$

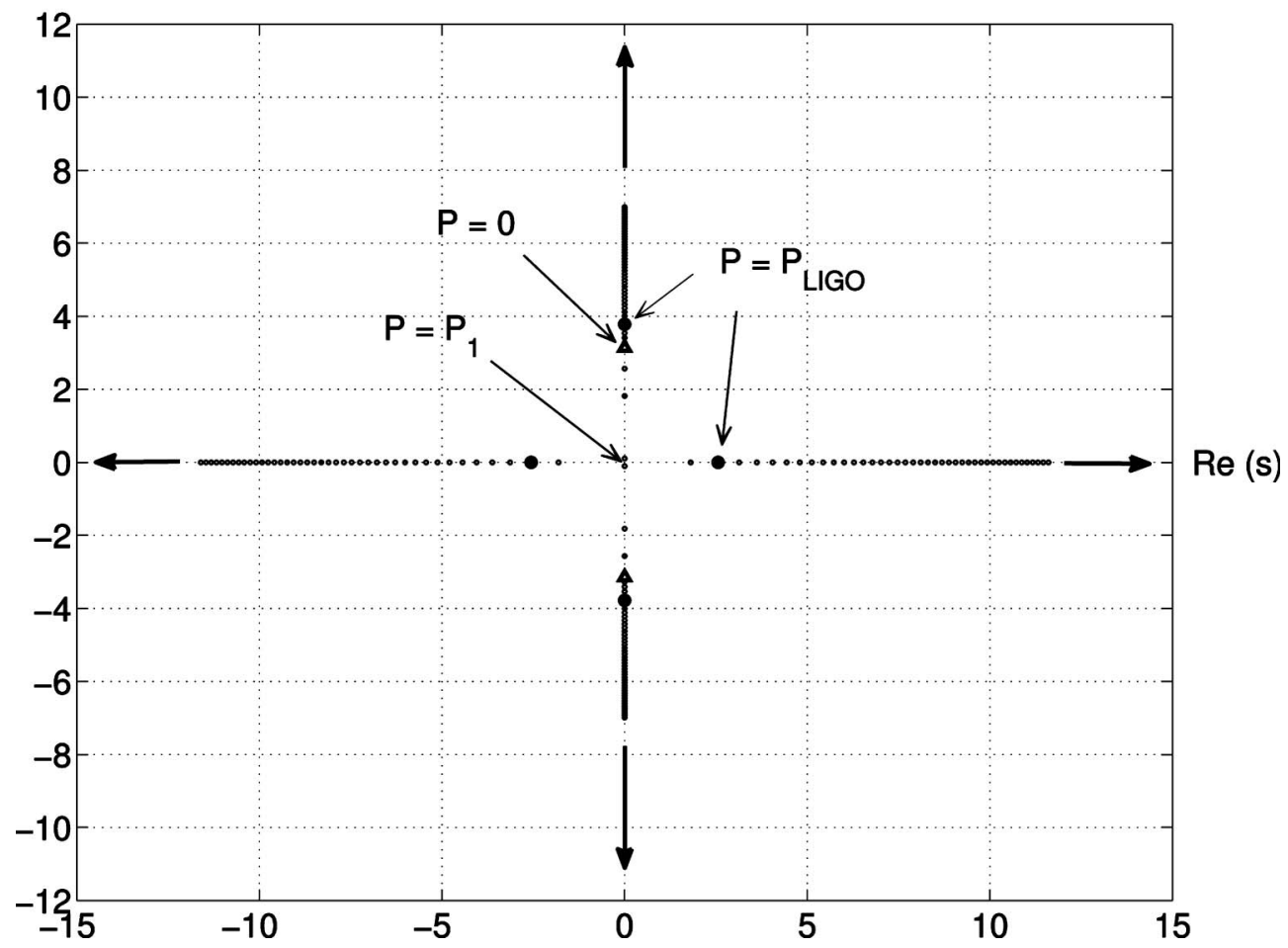

Fig. 5. Poles in the $s$ plane at various powers for the uncontrolled system. Triangles on the imaginary axis represent the locations of $\omega_{+}$, $\omega_{-}$, and $\omega_{z}$ at $P=0$ when they are all degenerate. As $P$ increases, $\omega_{+}$moves away from the origin while $\omega_{-}$moves toward the origin. After the pair for $\omega_{-}$hits the origin at $P=P_{1}$, the poles move along the real axis. The filled circles represent the locations of the poles at $P=P_{\text {LIGO }}$.

operating condition, in which the two arm cavities and the recycling cavity are resonant, and the complete control system is engaged. Then we injected an excitation signal into one of the wavefront sensors' control path that results in dithering the end test masses differentially. During the excitation, we monitored the optical lever signal of the end mirror in the $x$ arm and a signal that goes into the driver of the actuator attached to the mirror. Because the driver signal is proportional to the torque produced by the actuator, we were able to monitor the transfer function of the mirror angle $D$ to torque $C$ applied to the same mirror, up to some overall gain [see Fig. 6]. The transfer function is the direct analog of the one we introduced in the previous section, i.e., the response of the ETMX mirror angle to the torque applied to the mirror. In addition, we recorded signals from two points located just upstream $A$ and downstream $B$ of the excitation point in the wavefront sensors control loop [see Fig. 6]. This enables us to calculate the open loop transfer function of the control loop. We here call the two transfer functions $\operatorname{oplev}(s)$ and $\operatorname{olg}(s)$, respectively:

$$
\begin{gathered}
\operatorname{oplev}(s)=\frac{D}{C}, \\
\operatorname{olg}(s)=\frac{A}{B} .
\end{gathered}
$$

We made the measurement at three different input laser powers $(0.8 \mathrm{~W}, 4.0 \mathrm{~W}$, and $6.8 \mathrm{~W})$ [21]. In Fig. 7 , the open symbols show the results of our measurements of the oplev $(s)$ transfer function, while in Fig. 8 the open symbols show our measurements of $\operatorname{olg}(s)$.

There are several surprising features in Fig. 7. First, note that there is only one peak in the transfer function at each laser power, whose frequency decreases as the laser power goes up. This is something that we did not expect, based on the naive model (i.e., the model without any control loops). The predictions of the naive model, shown in Fig. 4, look qualitatively very different. Note also that, at the highest power level, a $180^{\circ}$ phase lead was observed. This was also surprising because it would usually indicate the system's instability.

\section{Mathematical Model}

These observations led us to construct a more complete model of the system that explicitly includes the dynamics of the angular control system. Figure 9 shows the block diagram. The most important parts of the control loop are the sensing matrix (labeled as $a, b, c$, and $d)$ and the control matrix $(B, C$, and $D)$. Each wavefront sensor measures a combination of the angles of the two mirrors (input and end mirrors); the signals are fed back to each mirror to minimize the deflection angle. The sensing matrix is measured separately in advance by shaking each mirror; the control matrix is determined by inverting the sensing matrix. Besides the wavefront sensors for global angular sensing, there is also a local loop called the 


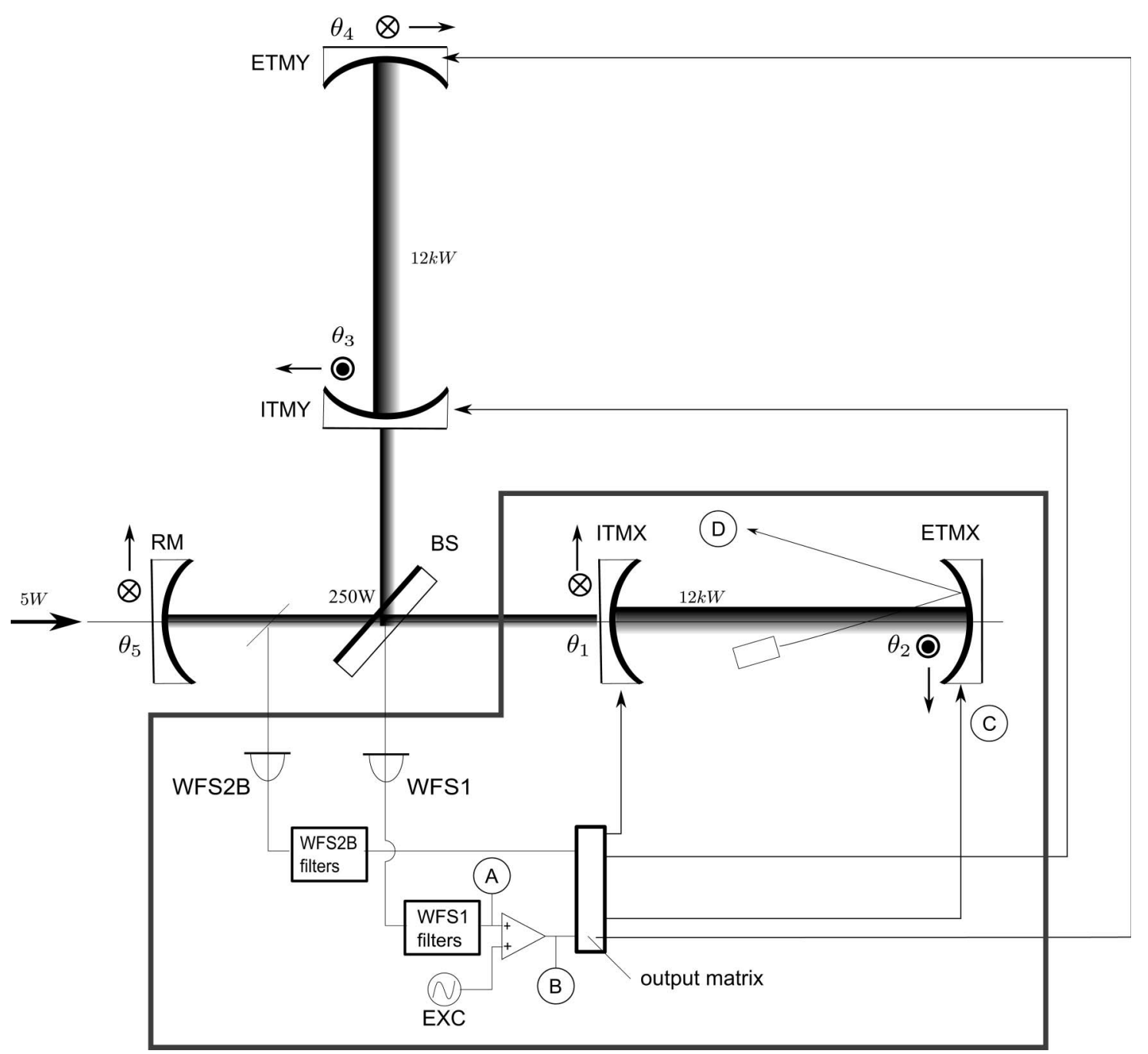

Fig. 6. Block diagram of our measurement scheme. An excitation signal is injected into the WFS1 path that dithers ETMX and ETMY differentially, i.e., $\Delta \theta_{\mathrm{ETM}}=\theta_{2}-\theta_{4}$. Both WFS1 and WFS2B are sensitive to the differential degrees of freedom $\left(\Delta \theta_{\mathrm{ETM}}\right.$ and $\left.\Delta \theta_{\text {ITM }}\right)$, but we focus on the contribution to the $x$ arm. The symbols next to each core optic show the rotation axes for pitch and yaw tilts.

optical lever for each mirror and a compensation loop to make the local loop invisible to the wavefront sensors. All filter banks associated with the two wavefront sensors are built into the model. The solid curves in Figs. 7 and 8 show the transfer functions calculated by our models. The different colors in the plots denote different cavity laser power levels in the model. They are blue for $1.6 \mathrm{~kW}$, green for $P=7.0 \mathrm{~kW}$, and red for $P=12.5 \mathrm{~kW}$. The agreement between the measurement and the model is very good. The only adjustable parameter in the model is the cavity laser power. Knowing that the full interferometer has many more degrees of freedom than are modeled here, such an agreement is especially gratifying.

(The cavity power levels chosen in the figures are those that best represent the observed behavior. They are consistent with our estimate of the power recycling factor; however, the cavity laser power is not simply proportional to the input laser power, but it is also affected by power-dependent thermal gradients inside the interferometer.)
There are several features worth noting in these transfer functions. First, we can now understand why there is only one peak observed in the optical lever transfer function. This resonance is associated with a pair of poles $s= \pm i \omega_{-}$, but the control loops make the transfer function much more complicated than Eq. (1). The reason why we only have one visible resonance is that with high loop gain as in initial LIGO, the pair of zeros $s= \pm i \omega_{z}$ and the other pair of poles $s= \pm i \omega_{+}$asymptotically approach the same value and cancel each other out.

Second, the frequency associated with the peak shifts toward lower frequencies as laser power goes up. This is understood as a radiation pressure effect, because the peak we observed corresponds to a pair of poles $s= \pm i \omega_{-}$and $\omega_{-}$decreases, as shown in Fig. 3 . The frequency does not go down as rapidly with increasing power as in the naive model, because the control system enhances the effective restoring force on the ITM.

Third, we can now understand why the phase of the optical lever transfer function at $6.8 \mathrm{~W}$ has a 

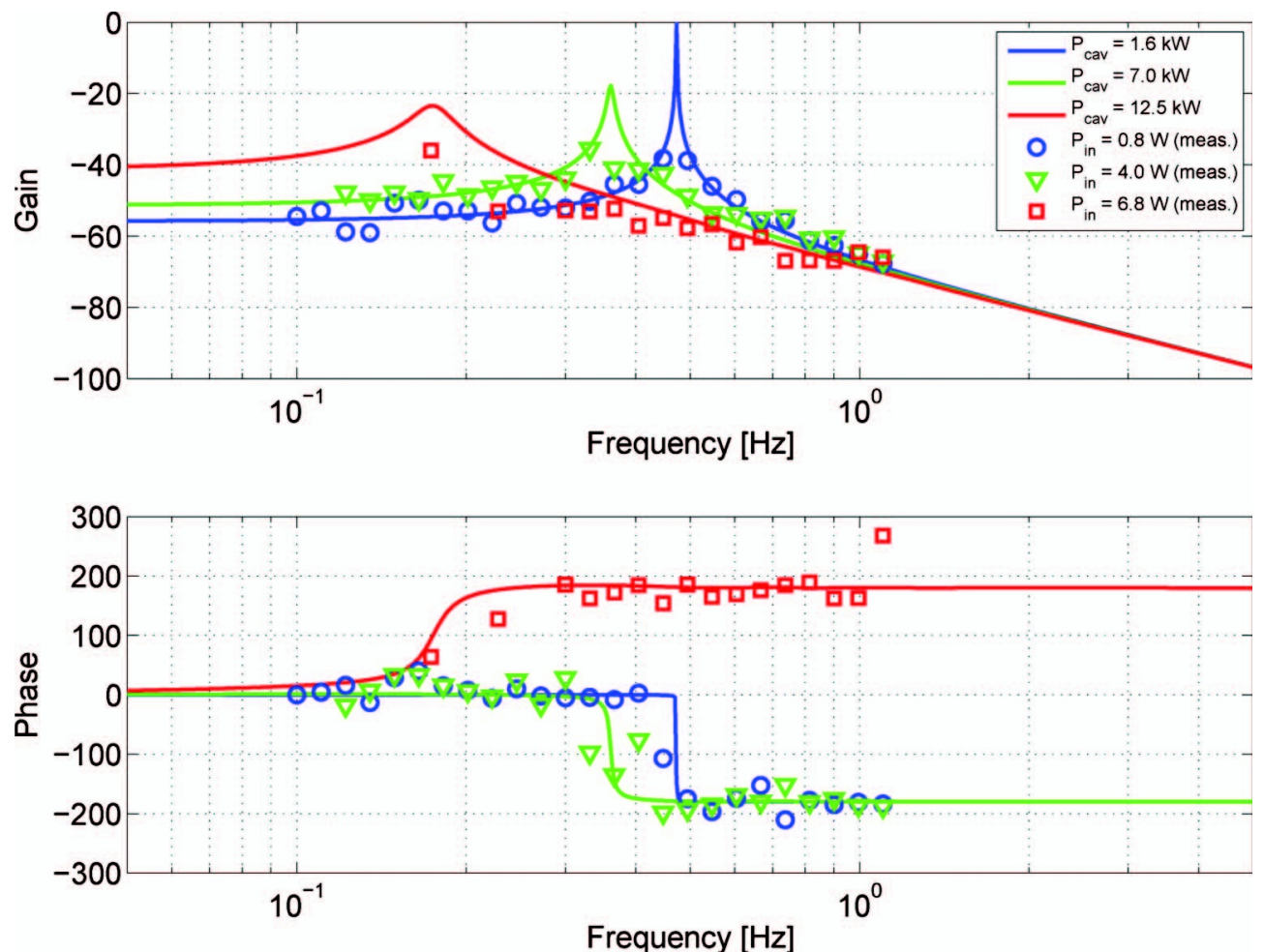

Fig. 7. Bode plot of the optical lever transfer function, oplev $(s)$. The input laser power levels are indicated as follows: $0.8 \mathrm{~W}$ (circles), $4.0 \mathrm{~W}$ (triangles), and $6.8 \mathrm{~W}$ (squares). The solid curves are the predictions of our mathematical model, including the effect of control loops, as described in Section $\underline{5}$.
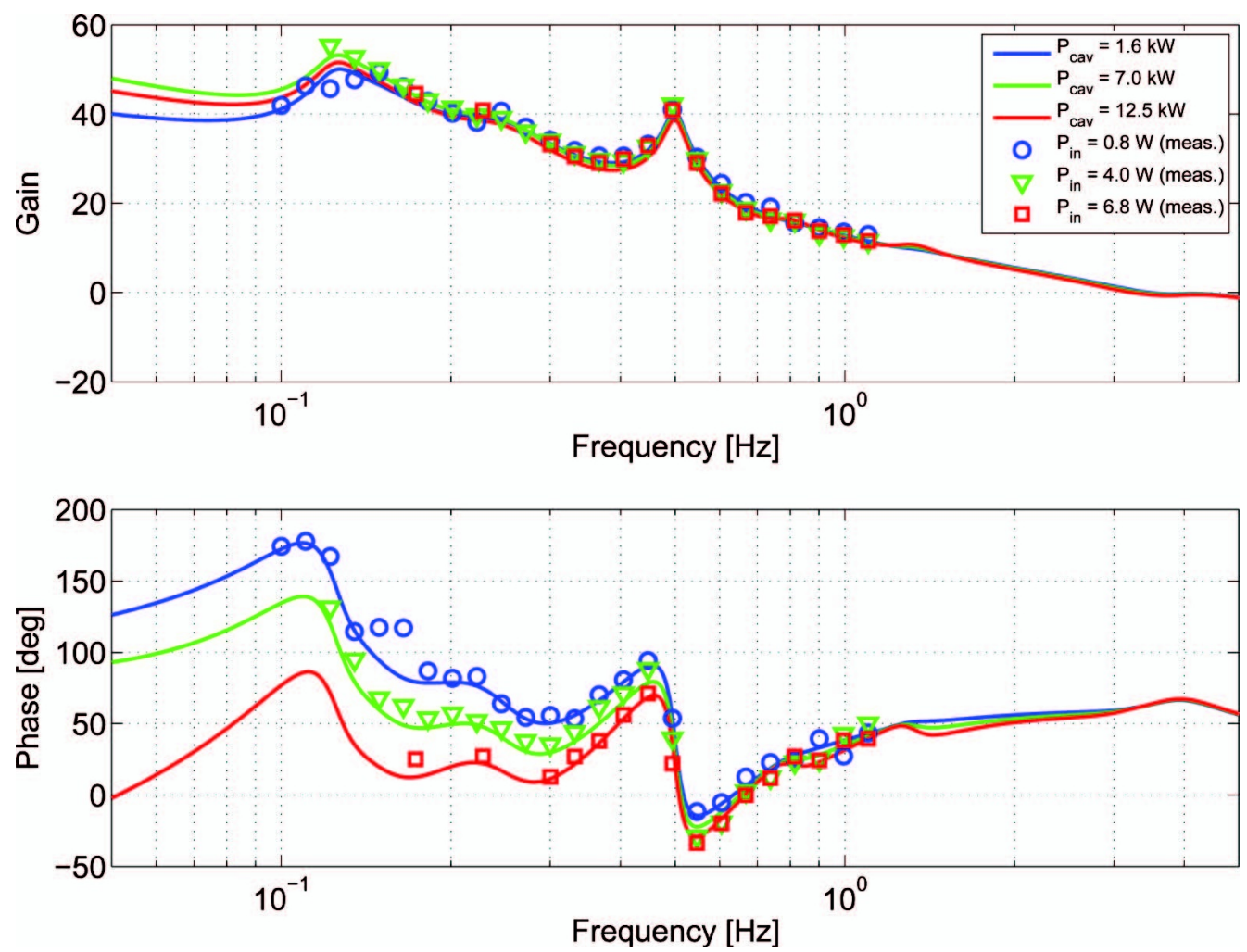

Fig. 8. Bode plot of the open loop transfer function, olg $(s)$. The solid curves are the predictions of our mathematical model, including the effect of control loops, as described in Section $\underline{5}$. 
$180^{\circ}$ phase lead. As noted above, under the influence of a strong control loop, the pair of poles associated with the stable mode cancels the pair of zeros and only the pair of unstable poles survives. In other words, when the control loop strongly suppresses the motion of the test masses, the in-loop transfer function oplev $(s)$ looks as if it were a single-mode unstable oscillator. This is clearly observed in both our measurement and the model as the phase advancement in the plot. This is evidence that instabilities due to radiation pressure, predicted by Sidles and Sigg, really exist in the interferometer and that the system successfully operates in the regime where the instability would occur in the absence of control.

Finally, we note that the radiation pressure effect is also observed in the phase of the open loop transfer function $\operatorname{olg}(s)$.

\section{Stability}

It is very important to know how much more power can be handled with the current control scheme for the upcoming higher power operation of the LIGO interferometers. In particular, an upgrade to initial
LIGO (called Enhanced LIGO) is now operational. It will have laser power roughly three times greater than initial LIGO with the same radius of curvature of the mirrors and an almost identical control system to that in initial LIGO.

When we discussed the stability of the system without control, a torque $T$ was applied to the system from "outside." However, with the control system in place, $T$ is no longer outside, but is included within the system. Therefore, in order to judge the stability of the whole system, we need to consider another torque $T^{\prime}$, which is located truly outside of the whole system [see Fig. 9]. So we cannot rely on the transfer function $\operatorname{oplev}(s)$ and Fig. 7 to judge the stability of the system with control. Instead, we will investigate the response of the system to such an outside torque:

$$
H^{\star}(s)=\frac{\theta_{2}}{T^{\prime}}
$$

Figure 10 shows how the system poles move in the $s$ plane as a function of the cavity laser power. (We calculate $H^{\star}(s)$ using our Simulink model. The

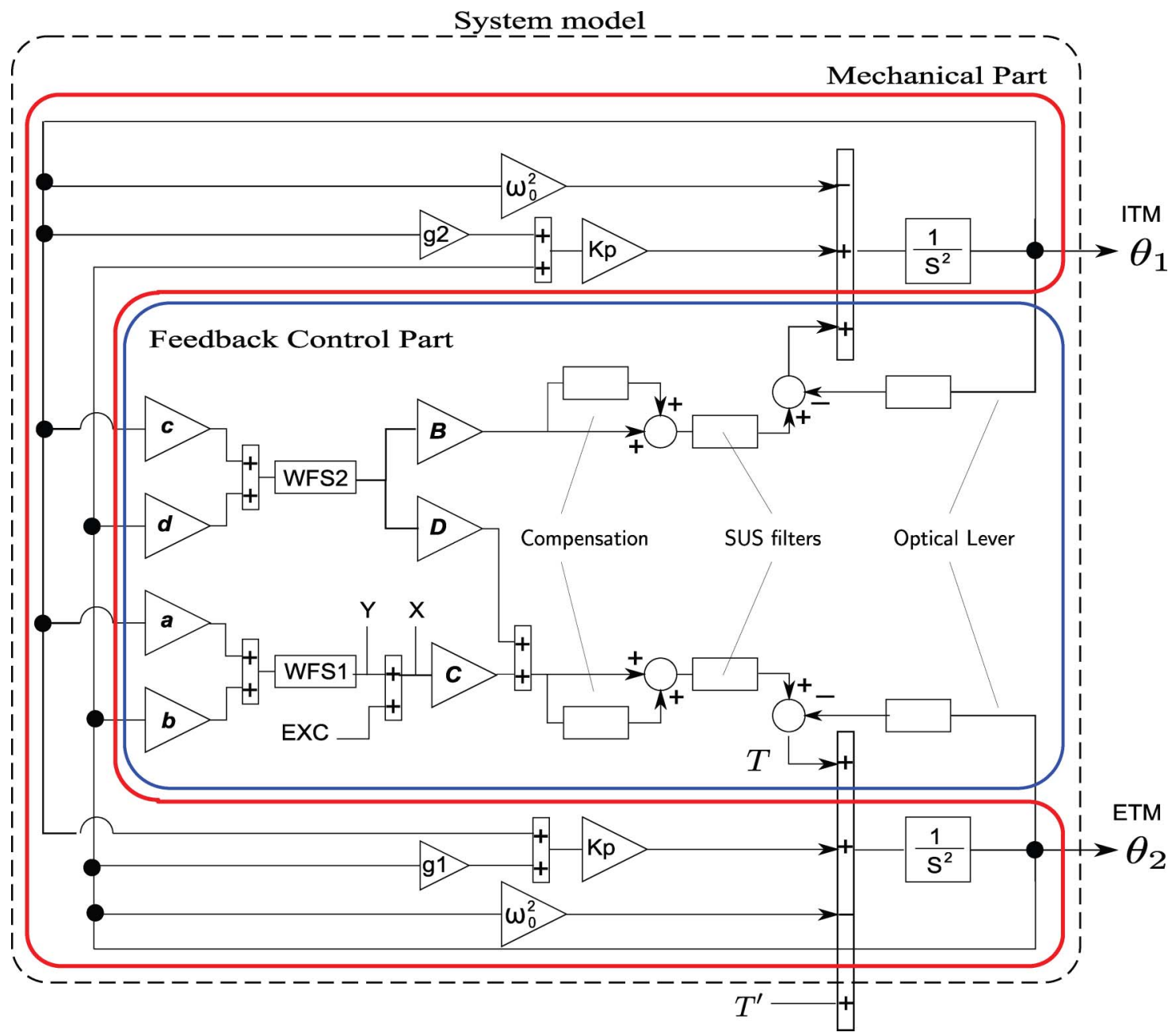

Fig. 9. (Color online) Block diagram of our model of an interferometer arm with angular feedback control. $a, b, c, d$, and $B, D, E$ are the sensing and control matrix, respectively. Rectangular blocks stand for filter banks. The suspension (SUS) filters are the ones related to the suspension system. The factor $K_{p}$ stands for the optical parameter $2 P L /\left[c I\left(1-g_{1} g_{2}\right)\right]$. 


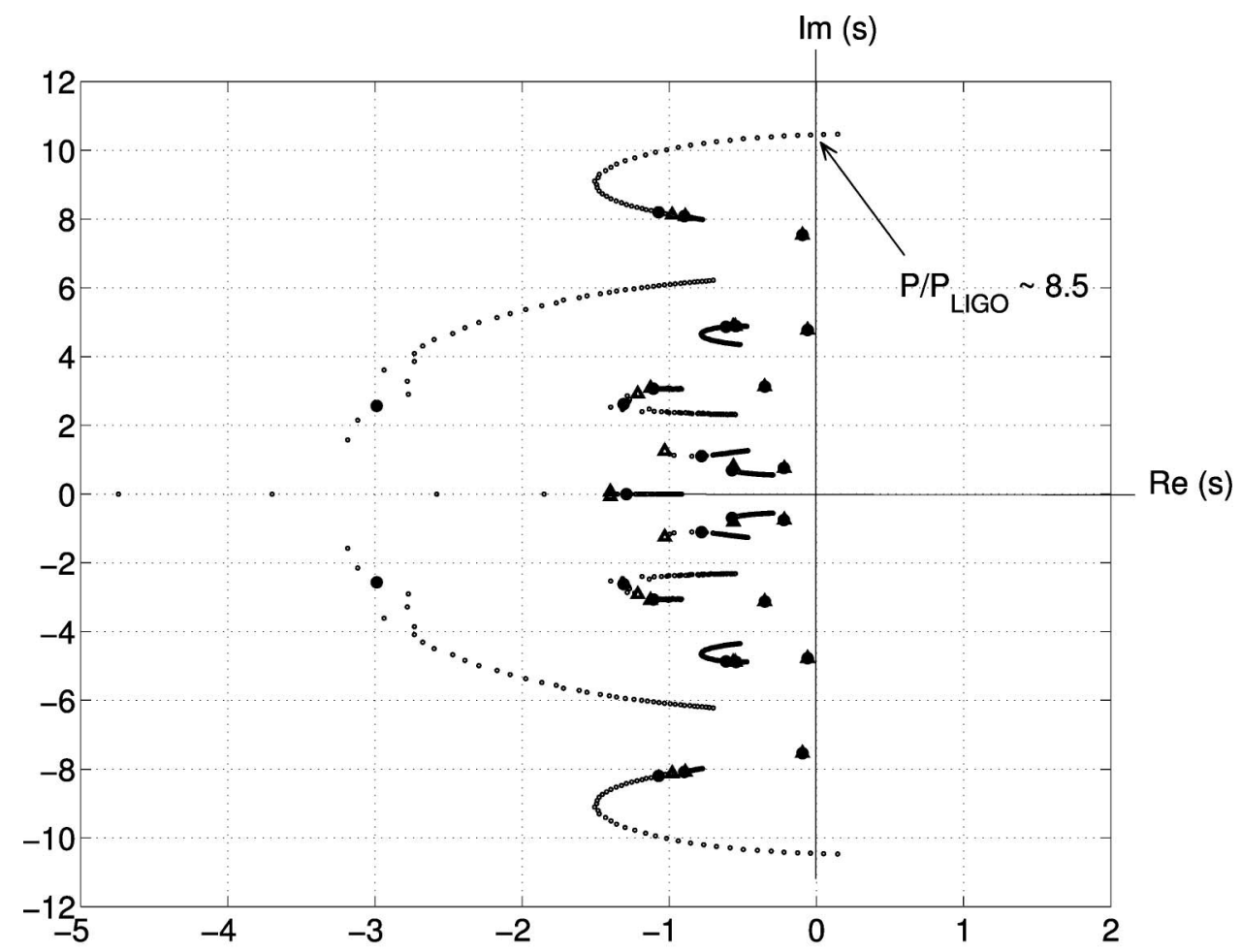

Fig. 10. Poles of the system with feedback control, with laser power from 0 to $110 \mathrm{~kW}$. The power increment between points is $2.5 \mathrm{~kW}$. Triangles and filled circles are for $P=0$ and $P=P_{\text {LIGO }}=12.5 \mathrm{~kW}$, respectively.

complexity of the model means that an algebraic expression would be difficult to write and is not likely to be illuminating.) We immediately notice the difference between Figs. 10 and 5 . Most of the poles are inserted by the filters and the rest of the complicated dynamics that represent a real interferometer [see Fig. 9]. The filled circles, which indicate the systems poles at the full laser power level for initial LIGO, are now all in the left half-plane, as expected. The dynamics of the system are dominated by the set of poles (the extreme top and bottom of the diagram) that will eventually cross into the right half-plane. (The other sets of poles near the imaginary axis are all canceled by zeros.)

According to our model, the system will remain stable until the cavity laser power reaches roughly eight times higher than that of initial LIGO. This analysis was based on the degrees of freedom associated with differential motion between the mirrors in the interferometer's two arms. The common mode degrees of freedom have lower unity gain frequencies in LIGO. This means that the full initial LIGO system would probably not remain stable at powers as high as are predicted in our analysis. A more complete analysis, including the common mode degrees of freedom, will be left for future work.

A new approach to this issue is being tested during the Enhanced LIGO commissioning process [22]. It uses a control scheme built around the stiff and soft modal basis [illustrated in Fig. 2]. We also note that Advanced LIGO will use arm cavities with negative $\mathrm{g}$ factors, to reduce its susceptibility to angular instability.
Other workers in the field have also made studies of the radiation-pressure-induced angular instability in optical cavities. Sakata has demonstrated the effect in a specially built apparatus at the National Astronomical Observatory of Japan [23]. Also, an Australian group observed the "soft" mode in an $80 \mathrm{~m}$ suspended optical cavity [24].

\section{Summary}

We observed the effect of radiation pressure on the angular control of the LIGO core optics at the $4 \mathrm{~km}$ interferometer at LIGO Hanford Observatory. This is the first measurement of this effect performed on a full gravitational-wave interferometer. Only one of two angular modes survives with feedback control because the other mode is suppressed when the control gain is large enough. A mathematical model was developed to understand the physics. It indicates that the system will remain stable at substantially higher power levels.

We are grateful to Sam Waldman for useful discussions. This research was supported in part by the National Science Foundation (NSF) under grant PHY-0600259. LIGO was constructed by the California Institute of Technology and the Massachusetts Institute of Technology with funding from the NSF and operates under cooperative agreement PHY0107417. This article has LIGO document number LIGO-P0900086.

\section{References}

1. B. C. Barish and R. Weiss, "LIGO and the detection of gravitational waves," Phys. Today 52, 44-50 (1999). 
2. B. P. Abbott, R. Abbott, R. Adhikari, P. Ajith, B. Allen, G. Allen, R. S. Amin, S. B. Anderson, W. G. Anderson, M. A. Arain, M. Araya, H. Armandula, P. Armor, Y. Aso, S. Aston, P. Aufmuth, C. Aulbert, S. Babak, P. Baker, S. Ballmer, C. Barker, D. Barker, B. Barr, P. Barriga, L. Barsotti, M. A. Barton, I. Bartos, R. Bassiri, M. Bastarrika, B. Behnke, M. Benacquista, J. Betzwieser, P. T. Beyersdorf, I. A. Bilenko, G. Billingsley, R. Biswas, E. Black, J. K. Blackburn, L. Blackburn, D. Blair, B. Bland, T. P. Bodiya, L. Bogue, R. Bork, V. Boschi, S. Bose, P. R. Brady, V. B. Braginsky, J. E. Brau, D. O. Bridges, M. Brinkmann, A. F. Brooks, D. A. Brown, A. Brummit, G. Brunet, A. Bullington, A. Buonanno, O. Burmeister, R. L. Byer, L. Cadonati, J. B. Camp, J. Cannizzo, K. C. Cannon, J. Cao, L. Cardenas, S. Caride, G. Castaldi, S. Caudill, M. Cavagli, C. Cepeda, T. Chalermsongsak, E. Chalkley, P. Charlton, S. Chatterji, S. Chelkowski, Y. Chen, N. Christensen, C. T. Y. Chung, D. Clark, J. Clark, J. H. Clayton, T. Cokelaer, C. N. Colacino, R. Conte, D. Cook, T. R. C. Corbitt, N. Cornish, D. Coward, D. C. Coyne, J. D. E. Creighton, T. D. Creighton, A. M. Cruise, R. M. Culter, A. Cumming, L. Cunningham, S. L. Danilishin, K. Danzmann, B. Daudert, G. Davies, E. J. Daw, D. DeBra, J. Degallaix, V. Dergachev, S. Desai, R. DeSalvo, S. Dhurandhar, M. Daz, A. Dietz, F. Donovan, K. L. Dooley, E. E. Doomes, R. W. P. Drever, J. Dueck, I. Duke, J.-C. Dumas, J. G. Dwyer, C. Echols, M. Edgar, A. Effler, P. Ehrens, E. Espinoza, T. Etzel, M. Evans, T. Evans, S. Fairhurst, Y. Faltas, Y. Fan, D. Fazi, H. Fehrmenn, L. S. Finn, K. Flasch, S. Foley, C. Forrest, N. Fotopoulos, A. Franzen, M. Frede, M. Frei, Z. Frei, A. Freise, R. Frey, T. Fricke, P. Fritschel, V. V. Frolov, M. Fyffe, V. Galdi, J. A. Garofoli, I. Gholami, J. A. Giaime, S. Giampanis, K. D. Giardina, K. Goda, E. Goetz, L. M. Goggin, G. Gonzlez, M. L. Gorodetsky, S. Goler, R. Gouaty, A. Grant, S. Gras, C. Gray, M. Gray, R. J. S. Greenhalgh, A. M. Gretarsson, F. Grimaldi, R. Grosso, H. Grote, S. Grunewald, M. Guenther, E. K. Gustafson, R. Gustafson, B. Hage, J. M. Hallam, D. Hammer, G. D. Hammond, C. Hanna, J. Hanson, J. Harms, G. M. Harry, I. W. Harry, E. D. Harstad, K. Haughian, K. Hayama, J. Heefner, I. S. Heng, A. Heptonstall, M. Hewitson, S. Hild, E. Hirose, D. Hoak, K. A. Hodge, K. Holt, D. J. Hosken, J. Hough, D. Hoyland, B. Hughey, S. H. Huttner, D. R. Ingram, T. Isogai, M. Ito, A. Ivanov, B. Johnson, W. W. Johnson, D. I. Jones, G. Jones, R. Jones, L. Ju, P. Kalmus, V. Kalogera, S. Kandhasamy, J. Kanner, D. Kasprzyk, E. Katsavounidis, K. Kawabe, S. Kawamura, F. Kawazoe, W. Kells, D. G. Keppel, A. Khalaidovski, F. Y. Khalili, R. Khan, E. Khazanov, P. King, J. S. Kissel, S. Klimenko, K. Kokeyama, V. Kondrashov, R. Kopparapu, S. Koranda, D. Kozak, B. Krishnan, R. Kumar, P. Kwee, P. K. Lam, M. Landry, B. Lantz, A. Lazzarini, H. Lei, M. Lei, N. Leindecker, I. Leonor, C. Li, H. Lin, P. E. Lindquist, T. B. Littenberg, N. A. Lockerbie, D. Lodhia, M. Longo, M. Lormand, P. Lu, M. Lubinski, A. Lucianetti, H. Lck, B. Machenschalk, M. MacInnis, M. Mageswaran, K. Mailand, I. Mandel, V. Mandic, S. Mrka, Z. Mrka, A. Markosyan, J. Markowitz, E. Maros, I. W. Martin, R. M. Martin, J. N. Marx, K. Mason, F. Matichard, L. Matone, R. A. Matzner, N. Mavalvala, R. McCarthy, D. E. McClelland, S. C. McGuire, M. McHugh, G. McIntyre, D. J. A. McKechan, K. McKenzie, M. Mehmet, A. Melatos, A. C. Melissinos, D. F. Menndez, G. Mendell, R. A. Mercer, S. Meshkov, C. Messenger, M. S. Meyer, J. Miller, J. Minelli, Y. Mino, V. P. Mitrofanov, G. Mitselmakher, R. Mittleman, O. Miyakawa, B. Moe, S. D. Mohanty, S. R. P. Mohapatra, G. Moreno, T. Morioka, K. Mors, K. Mossavi, C. MowLowry, G. Mueller, H. Miller-Ebhardt, D. Muhammad, S. Mukherjee, H. Mukhopadhyay, A. Mullavey, J. Munch, P. G. Murray, E. Myers, J. Myers, T. Nash, J. Nelson, G. Newton, A. Nishizawa, K. Numata, J. O'Dell, B. O'Reilly, R. O'Shaughnessy, E. Ochsner, G. H. Ogin, D. J. Ottaway, R. S.
Ottens, H. Overmier, B. J. Owen, Y. Pan, C. Pankow, M. A. Papa, V. Parameshwaraiah, P. Patel, M. Pedraza, S. Penn, A. Perraca, V. Pierro, I. M. Pinto, M. Pitkin, H. J. Pletsch, M. V. Plissi, F. Postiglione, M. Principe, R. Prix, L. Prokhorov, O. Punken, V. Quetschke, F. J. Raab, D. S. Rabeling, H. Radkins, P. Raffai, Z. Raics, N. Rainer, M. Rakhmanov, V. Raymond, C. M. Reed, T. Reed, H. Rehbein, S. Reid, D. H. Reitze, R. Riesen, K. Riles, B. Rivera, P. Roberts, N. A. Robertson, C. Robinson, E. L. Robinson, S. Roddy, C. Rver, J. Rollins, J. D. Romano, J. H. Romie, S. Rowan, A. Rdiger, P. Russell, K. Ryan, S. Sakata, L. Sancho de la Jordana, V. Sandberg, V. Sannibale, L. Santamara, S. Saraf, P. Sarin, B. S. Sathyaprakash, S. Sato, M. Satterthwaite, P. R. Saulson, R. Savage, P. Savov, M. Scanlan, R. Schilling, R. Schnabel, R. Schofield, B. Schulz, B. F. Schutz, P. Schwinberg, J. Scott, S. M. Scott, A. C. Searle, B. Sears, F. Seifert, D. Sellers, A. S. Sengupta, A. Sergeev, B. Shapiro, P. Shawhan, D. H. Shoemaker, A. Sibley, X. Siemens, D. Sigg, S. Sinha, A. M. Sintes, B. J. J. Slagmolen, J. Slutsky, J. R. Smith, M. R. Smith, N. D. Smith, K. Somiya, B. Sorazu, A. Stein, L. C. Stein, S. Steplewski, A. Stochino, R. Stone, K. A. Strain, S. Strigin, A. Stroeer, A. L. Stuver, T. Z. Summerscales, K.-X. Sun, M. Sung, P. J. Sutton, G. P. Szokoly, D. Talukder, L. Tang, D. B. Tanner, S. P. Tarabrin, J. R. Taylor, R. Taylor, J. Thacker, K. A. Thorne, A. Thring, K. V. Tokmakov, C. Torres, C. Torrie, G. Traylor, M. Trias, D. Ugolini, J. Ulmen, K. Urbanek, H. Vahlbruch, M. Vallisneri, C. Van Den Broeck, M. V. van der Sluys, A. A. van Veggel, S. Vass, R. Vaulin, A. Vecchio, J. Veitch, P. Veitch, C. Veltkamp, A. Villar, C. Vorvick, S. P. Vyachanin, S. J. Waldman, L. Wallace, R. L. Ward, A. Weidner, M. Weinert, A. J. Weinstein, R. Weiss, L. Wen, S. Wen, K. Wette, J. T. Whelan, S. E. Whitcomb, B. F. Whiting, C. Wilkinson, P. A. Willems, H. R. Williams, L. Williams, B. Willke, I. Wilmut, L. Winkelmann, W. Winkler, C. C. Wipf, A. G. Wiseman, G. Woan, R. Wooley, J. Worden, W. Wu, I. Yakushin, H. Yamamoto, Z. Yan, S. Yoshida, M. Zanolin, J. Zhang, L. Zhang, C. Zhao, N. Zotov, M. E. Zucker, H. zur Mhlen, and J. Zweizig, "LIGO: the laser interferometer gravitational-wave observatory," Rep. Prog. Phys. 72, 076901 (2009).

3. S. J. Waldman and the LIGO Scientific Collaboration, "Status of LIGO at the start of the fifth science run," Class. Quantum Grav. 23, S653-S660 (2006).

4. D. Sigg and the LIGO Scientific Collaboration, "Status of the LIGO detectors," Class. Quantum Grav. 23, S51-S56 (2006).

5. Keita Kawabe and the LIGO Scientific Collaboration), "Status of LIGO," J. Phys. 120, 032003 (2008).

6. H. Grote and the LIGO Scientific Collaboration "The status of GEO 600," Class. Quantum Grav. 25, 114043 (2008).

7. F. Acernese, M. Alshourbagy, P. Amico, F. Antonucci, S. Aoudia, P. Astone, S. Avino, L. Baggio, G. Ballardin, F. Barone, L. Barsotti, M. Barsuglia, T. S. Bauer, S. Bigotta, S. Birindelli, M. A. Bizouard, C. Boccara, F. Bondu, L. Bosi, S. Braccini, C. Bradaschia, A. Brillet, V. Brisson, D. Buskulic, G. Cagnoli, E. Calloni, E. Campagna, F. Carbognani, F. Cavalier, R. Cavalieri, G. Cella, E. Cesarini, E. Chassande-Mottin, A.-C. Clapson, F. Cleva, E. Coccia, C. Corda, A. Corsi, F. Cottone, J.-P. Coulon, E. Cuoco, S. D. Antonio, A. Dari, V. Dattilo, M. Davier, R. De Rosa, M. Del Prete, L. Di Fiore, A. Di Lieto, M. Di Paolo Emilio, A. Di Virgilio, M. Evans, V. Fafone, I. Ferrante, F. Fidecaro, I. Fiori, R. Flaminio, J.-D. Fournier, S. Frasca, F. Frasconi, L. Gammaitoni, F. Garufi, E. Genin, A. Gennai, A. Giazotto, L. Giordano, V. Granata, C. Greverie, D. Grosjean, G. Guidi, S. Hamdani, S. Hebri, H. Heitmann, P. Hello, D. Huet, S. Kreckelbergh, P. La Penna, M. Laval, N. Leroy, N. Letendre, B. Lopez, M. Lorenzini, V. Loriette, G. Losurdo, J.-M. Mackowski, E. Majorana, C. N. Man, M. Mantovani, F. Marchesoni, F. Marion, J. Marque, F. Martelli, 
A. Masserot, F. Menzinger, L. Milano, Y. Minenkov, C. Moins, J. Moreau, N. Morgado, S. Mosca, B. Mours, I. Neri, F. Nocera, G. Pagliaroli, C. Palomba, F. Paoletti, S. Pardi, A. Pasqualetti, R. Passaquieti, D. Passuello, F. Piergiovanni, L. Pinard, R. Poggiani, M. Punturo, P. Puppo, P. Rapagnani, T. Regimbau, A. Remillieux, F. Ricci, I. Ricciardi, A. Rocchi, L. Rolland, R. Romano, P. Ruggi, G. Russo, S. Solimeno, A. Spallicci, M. Tarallo, R. Terenzi, A. Toncelli, M. Tonelli, E. Tournefier, F. Travasso, C. Tremola, G. Vajente, J. F. J. van den Brand, S. van der Putten, D. Verkindt, F. Vetrano, A. Vicer, J.-Y. Vinet, H. Vocca, and M. Yvert, "VIRGO status," Class. Quantum Grav. 25, 184001 (2008).

8. “Advanced LIGO reference design," LIGO Tech. Rep. M060056 (Laser Interferometer Gravitational-Wave Observatory, 2007)

9. R. W. P. Drever, J. Hough, A. J. Munley, S.-A. Lee, R. Spero, S. E. Whitcomb, H. Ward, G. M. Ford, M. Hereld, N. A. Robertson, I. Kerr, J. R. Pugh, G. P. Newton, B. Meers, E. D. Brook III, and Y. Gursel, "Gravitational wave detectors using laser interferometers and optical cavities," Quantum Optics, Experimental Gravitation and Measurement Theory, P. Meystre and M. O. Scully, eds. (Plenum, 1983), pp. 503-524.

10. T. Corbitt, D. Ottaway, E. Innerhofer, J. Pelc, and N. Mavalvala, "Measurement of radiation-pressure-induced optomechanical dynamics in a suspended Fabry-Perot cavity," Phys. Rev. A 74, 021802(R) (2006).

11. J. A. Sidles and D. Sigg, "Optical torques in suspended Fabry-Perot interferometers," Phys. Lett. A 354, 167-172 (2006).

12. S. Solimeno, F. Barone, C. De Lisio, L. Di Fiore, L. Milano, and G. Russo, "Fabry-Perot resonators with oscillating mirrors," Phys. Rev. A 43, 6227-6240 (1991).

13. MathWorks, MATLAB and Simulink.

14. P. Fritschel, G. González, N. Mavalvala, D. Shoemaker, D. Sigg, and M. Zucker, "Alignment of an interferometric gravitational wave detector," Appl. Opt. 37, 6734-6747 (1998).

15. Y. Hefetz, N. Mavalvala, and D. Sigg, "Principles of calculating alignment signals in complex resonant optical interferometers," J. Opt. Soc. Am. B 14, 1597-1605 (1997).

16. D. Sigg and N. Mavalvala, "Principles of calculating the dynamical response of misaligned complex resonant optical interferometers," J. Opt. Soc. Am. A 17, 1642-1649 (2000).

17. P. Fritschel, R. Bork, G. González, N. Mavalvala, D. Ouimette, H. Rong, D. Sigg, and M. Zucker, "Readout and control of a power-recycled interferometric gravitational-wave antenna," Appl. Opt. 40, 4988-4998 (2001).

18. D. Z. Anderson, "Alignment of resonant optical cavities," Appl. Opt. 23, 2944-2949 (1984).

19. E. Morrison, B. J. Meers, D. I. Robertson, and H. Ward, "Automatic alignment of optical interferometers," Appl. Opt. 33, 5041-5049 (1994).

20. S. Kawamura and M. E. Zucker, "Mirror-orientation noise in a Fabry-Perot interferometer gravitational wave detector," Appl. Opt. 33, 3912-3918 (1994).

21. E. Hirose and K. Kawabe, "A study of radiation pressure effect on ASC in H1 and H2," LIGO-G070118-00-Z (Laser Interferometer Gravitational-Wave Observatory, 2007)

22. L. Barsotti and M. Evans, "Modeling of alignment sensing and control for enhanced LIGO," LIGO-T080186-01-I (Laser Interferometer Gravitational-Wave Observatory, 2008).

23. S. Sakata, "A study of angular instability due to radiation pressure in LIGO gravitational wave detector," Ph.D. thesis (Ochanomizu University, 2008).

24. Y. Fan, L. Merrill, C. Zhao, L. Ju, D. Blair, B. Slagmolen, D. Hosken, A. Brooks, P. Veitch, D. Mudge, and J. Munch, "Observation of optical torsional stiffness in a high optical power cavity,” Appl. Phys. Lett. 94, 081105 (2009). 